\title{
Intranasal squamous cell carcinoma in a renal transplant recipient on long term immunosuppression
}

\author{
A. Pace-Balzan and M.S. Timms \\ Department of Otolaryngology, Manchester Royal Infirmary, Oxford Road, Manchester M139WL, UK.
}

\begin{abstract}
Summary: An increased risk of developing malignant disease after renal transplantation and immunosuppression with azathioprine and corticosteroids is well documented. We report a case of intranasal carcinoma occurring in a renal transplant recipient. The possible role of immunosuppression on the development and on the outcome of treatment of this tumour is discussed.
\end{abstract}

\section{Introduction}

We report a case of carcinoma of the nasal cavity arising in a young woman on long term immunosuppressive therapy with azathioprine and prednisolone following successful cadaver renal transplant. To our knowledge an intranasal carcinoma has not been reported previously in a transplant recipient.

\section{Case report}

A 43 year old Caucasian woman presented with an 8 weeks' history of an enlarging painless swelling over the left side of her nose, accompanied by left sided nasal obstruction and blood-stained discharge. She was otherwise symptom-free. She had undergone a successful cadaver renal transplant 7 years previously and had been maintained on azathioprine $100 \mathrm{mg} /$ day and prednisolone in varying dosage since then. On examination the left side of the nose was deformed by a firm $2 \mathrm{~cm}$ swelling which was fixed to the nasal skeleton (Figure 1). The overlying skin was normal and not attached to the swelling. On anterior rhinoscopy there was a swelling protruding into the atrium of the left nasal cavity with ulceration of the overlying mucosa. The rest of the examination, in particular examination of the rest of the head and neck, was normal. Radiology and computerized tomography (CT) showed no involvement of the paranasal sinuses. An intranasal biopsy of the lesion was reported as showing poorly differentiated squamous cell carcinoma. The patient underwent a radical course of radiotherapy which resulted in resolution of the mass. The swelling recurred 4 months after radiotherapy, with $\mathrm{X}$-rays and CT scan now showing a very extensive

Correspondence: A. Pace-Balzan, F.R.C.S

Accepted: 10 April 1987 tumour which filled the left ethmoid sinus and extended into the sphenoid sinus and into the left orbit. There was still no lymph node involvement and the patient underwent a left maxillectomy and ethmoidectomy with orbital exenteration. Six months postoperatively the tumour recurred locally and extended intracranially along the optic nerve. The patient was maintained on azathioprine and prednisolone throughout.

\section{Discussion}

The increased incidence of neoplasia in organ transplant recipients on long term immunosuppressive therapy with azathioprine is well documented. In 1975 Penn ${ }^{1}$ reported an incidence of malignant disease in a series of transplant patients of $5.6 \%$ which he calculated to be approximately 100 times the incidence observed in the general population in the same age range. A geographical variation in incidence rates has been observed, with a range of $1.6 \%$ in a European series $^{2}$ to $24 \%$ in an Australian series. ${ }^{3}$

The incidence of malignant disease in transplant recipients has also been shown to increase steadily with the interval from transplantation. An incidence of cancer in transplant recipients surviving beyond one, five and nine years of $23 \%, 39 \%$ and $44 \%$ respectively has been reported. ${ }^{4}$

The commonest tumours encountered in immunosuppressed transplant recipients are cancer of the skin and lip which are 7 times more common than in the general population, and tumours of the lymphoreticular system particularly reticulum cell sarcoma which is $\mathbf{3 5 0}$ times more common than in the general

(C) The Fellowship of Postgraduate Medicine, 1987 


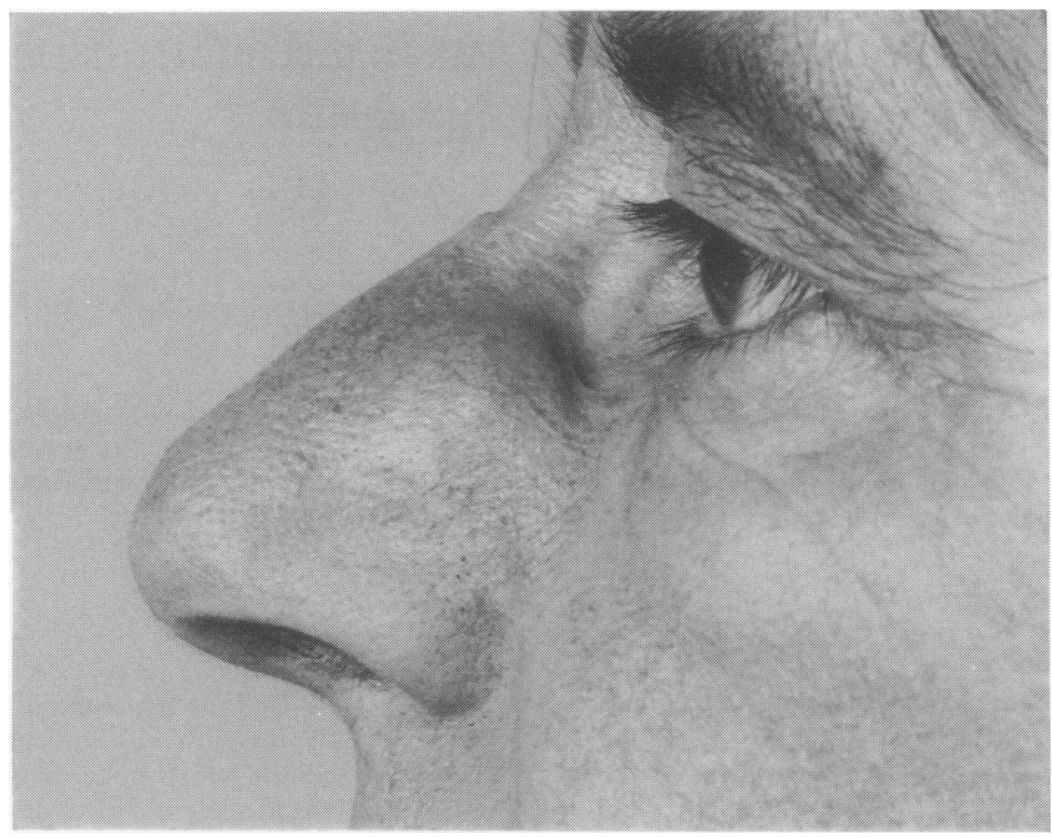

Figure 1 Swelling deforming left side of nose. The overlying skin is normal.

population. ${ }^{5}$ However, while great emphasis has been placed on these tumours, other cancers are occurring with increased frequency in the young transplant population and it seems likely that most, if not all, cancers will be found to occur with increased frequency in transplant recipients. ${ }^{4}$ In spite of all the evidence for this increased incidence of malignant disease in transplant patients it is difficult to be certain of a causal association in any individual patient. Such an association is very likely in this case of squamous cell carcinoma of the nasal cavity in a young woman appearing 80 months after renal transplantation, bearing in mind that cancer of the nose and sinuses is uncommon, accounting for $0.2 \%-0.8 \%$ of all cancers and $3 \%$ of all cancer of the upper aerodigestive tract. These tumours normally occur more commonly in men and the majority of patients are over 50 years of age at the time of diagnosis. ${ }^{6}$

Another point about malignant disease in transplant patients, apart from the increased incidence, concerns the behaviour of some of the tumours encountered. Reporting on squamous cell carcinomas of skin Sheil et al. ${ }^{4}$ comment on the increased aggressiveness of these tumours, most being multiple and having a marked tendency for recurrence and metastasis. The neoplasm in our patient was particularly aggressive producing very extensive recurrence within 4 months of radical radiotherapy. In the general population $35 \%$ of women with squamous cell carci noma of the nasal cavity survive 5 years. ${ }^{7}$ The aggressive behaviour of the tumour in this particular case raises the questions of whether continuation of the immunosuppressive therapy contributed to the aggressiveness of this tumour, and whether immunosuppressive therapy should be stopped when a malignant tumour appears. Raising this latter point Penn ${ }^{5}$ states that experience in this area is limited but that in the case of Kaposi's sarcoma the evidence suggests that alteration of immunosuppression is worthwhile. Reporting on squamous cell carcinomas of skin, Sheil et al. ${ }^{3}$ state that withdrawal of azathioprine therapy in four of their patients with generalized carcinomatous changes decreased the rate of progression of the tumour but resulted in graft rejection in from 4 to 8 months in all the patients.

The development and behaviour of the neoplasm in this particular case would seem to be related to the immunosuppressive therapy. In searching the literature for guidelines on the management of neoplasms in transplant patients, we found the evidence regarding the difficult question of whether or not to stop immunosuppressive therapy inconclusive. It is hoped that by highlighting this difficulty, some guidelines on this aspect of the management of transplant recipients with malignant disease may eventually appear. 


\section{Acknowledgements}

We thank Mr R.W.G. Johnson F.R.C.S. and Mr R.T. Ramsden F.R.C.S for allowing us to report this case and thank Miss I.M. Chetwood for secretarial services.

\section{References}

1. Penn, $I$. The incidence of malignancies in transplant recipients. Transplant Proc 1975, VII: 323-326.

2. Jacobs, C., Brunner, F.P., Brynger, H. et al. Malignant diseases in patients treated by dialysis and transplantation in Europe. Transplantation 1981, XIII: 729-732.

3. Sheil, A.G.R. Mahoney, J.F, Horvath, J.S. et al. Cancer following successful cadaveric donor renal transplantation. Transplant Proc 1981, XIII: 733-735.

4. Sheil, A.G.R., Mahoney, J.F., Horvath, J.S. et al. Cancer following renal transplantation. Aust NZ J Surg 1979, 49: 617-620.
5. Penn, I. Tumour incidence in human allograft recipients. Transplant Proc 1979, XI: 1047-1051.

6. Batsakis, J.G., Rice, D.H. \& Solomon, A.R. The pathology of head and neck tumours: squamous and mucousgland carcinomas of the nasal cavity, paranasal sinuses, and larynx, part 6. Head Neck Surg 1980, 2: 497-508.

7. Robin, P.E., Powell, D.J. \& Stansbie, J.M. Carcinoma of the nasal cavity and paranasal sinuses: incidence and presentation of different histological types. Clin Otolaryngol 1979, 4: 431-456. 